\title{
T3NS: three-legged tree tensor network states
}

\author{
Klaas Gunst, ${ }^{*, \dagger, \ddagger}$ Frank Verstraete, ${ }^{\ddagger}$, Sebastian Wouters, ${ }^{\S}$ Örs Legeza, $\|$ and \\ Dimitri Van Neck ${ }^{\dagger}$ \\ $\dagger$ †enter for Molecular Modeling, Ghent University, Technologiepark 903, 9052 Zwijnaarde, \\ Belgium \\ $\ddagger$ Department of Physics and Astronomy, Ghent University, Krijgslaan 281, S9, B-9000 \\ Ghent, Belgium \\ IVienna Center for Quantum Technology, University of Vienna, Boltzmanngasse 5, 1090 \\ Vienna, Austria \\ $\S$ Brantsandpatents, Pauline van Pottelsberghelaan 24, 9051 Sint-Denijs Westrem (Ghent), \\ Belgium \\ ||Strongly Correlated Systems "Lendület" Research group, Wigner Research Centre for \\ Physics, H-1525, Budapest, Hungary \\ E-mail: Klaas.Gunst@UGent.be
}

\section{Abstract}

We present a new variational tree tensor network state (TTNS) ansatz, the three-legged tree tensor network state (T3NS). Physical tensors are interspersed with branching tensors. Physical tensors have one physical index and at most two virtual indices, as in the matrix product state (MPS) ansatz of the density matrix renormalization group (DMRG). Branching tensors have no physical index, but up to three virtual indices. In this way, advantages of DMRG, in particular a low computational cost and a simple implementation of symmetries, are combined with advantages of TTNS, namely incorporating more entanglement. Our code is capable of simulating quantum chemical Hamiltonians, and we present several proof-of-principle calculations on LiF, $\mathrm{N}_{2}$ and the bis( $\mu$-oxo) and $\mu-\eta^{2}: \eta^{2}$ peroxo isomers of $\left[\mathrm{Cu}_{2} \mathrm{O}_{2}\right]^{2+}$.

\section{Introduction}

Since its formulation in 1992 by S. White, ${ }^{112}$ the density matrix renormalization group (DMRG) method has quickly proved its usefulness in the simulation of strongly correlated quantum systems, both in condensed matter physics and theoretical chemistry. While initially applied on systems with local Hamiltonians, it didn't take long before it was applied successfully on systems with long-range interactions, like in momentum space representation $(\mathrm{k}-\mathrm{DMRG})^{\underline{3}}$ and quantum chemistry (QC-DMRG). 4

Later on, it was found that DMRG corresponded with the variational optimization of a particular wave function, the matrix product state (MPS). [5]6 In an MPS, the state is represented by a linear chain of tensors, providing a very efficient parametrization of states respecting the area law for entanglement in 1D systems. This explained the high efficiency of DMRG for the description of ground states of one-dimensional non-critical local Hamiltonians. It also clarified the connection with quantum information theory, and paved the way to more advanced wave functions, the so-called tensor network states (TNS).

Changing from one dimensional MPSs to other types of TNSs allowed efficient descriptions of entanglement in higher dimensions and in critical systems. Because of this, these methods are gaining more and more momentum, 
especially in condensed matter physics. Notable examples of more general TNSs are, for example, the projected entangled pair states $(\mathrm{PEPS})^{7}$ and the multi-scale entanglement renormalization ansatz (MERA).

In quantum chemistry, other tensor network states have also been studied. Notable examples are tree tensor network states (TTNS), $\underline{911}$ which are the subject of this paper, completegraph tensor network states (CGTNS) ${ }^{12}$ and self-adaptive tensor network states (SATNS). 13 However, the MPS is still the preferred tensor network for quantum chemistry, although its one-dimensional nature is far from ideal save for linear molecules. Due to the high efficiency and stability of the algorithm and the relative ease of implementing $S U(2)$-symmetry, the suboptimal entanglement representation can be lifted by increasing the virtual bond dimension enough.

In this paper, we use the three-legged tree tensor network state (T3NS), a subclass of TTNSs. We believe it is able to represent the entanglement of a general molecule more accurately while still being computationally efficient. In this subclass, we also expect that the implementation of $S U(2)$-symmetry will be no more difficult than for DMRG, which is an important prerequisite for obtaining a highly accurate and efficient algorithm in quantum chemistry.

The paper is structured as follows. In section 2, general tree tensor networks are briefly explained and an overview is given of previous research in TTNS for quantum chemistry (QCTTNS). The T3NS is defined in subsection 2.1. followed by a short explanation of the fermionic sign handling in subsection 2.2. The complexity of the algorithm, some of the most intensive steps in the algorithm and factors that influence speed and accuracy are discussed in subsections 2.3 and 2.4. In section 3, calculations for different quantum mechanical systems are discussed using T3NS. Summary and conclusions are provided in section 4 .

This paper is meant for the reader already familiar with TNS, DMRG and more particularly QC-DMRG. For a thorough study of these subjects we refer to refs. 1, 2,4,14, 24.

\section{Tree Tensor Networks}

The TTNS is a natural extension of the MPS ansatz which is used in DMRG. While the MPS wave function can be depicted as a linear chain of tensors, the TTNS ansatz allows branching of the network. The TTNS ansatz is the most general tensor network state without any loops. It allows an exact treatment from the mathematical point of view as higher order singular value decomposition (HOSVD) can be applied. By using this ansatz, a better representation of the entanglement topology of the system is expected as compared to the MPS, since component tensors can have an arbitrary order.

A substantial advantage of TTNS is that at a finite bond dimension it is able to capture algebraically decaying correlation functions. This in contrast to DMRG which is only able to represent exponentially decaying corre-

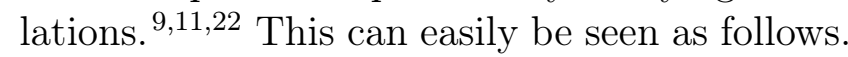
Imagine we start from one central tensor and we radially expand the TTNS with a fixed coordination number $z$ (i.e. the maximum number of virtual bonds of a tensor in the TTNS). The number of sites $L$ in function of the number of layers $Y$ is

$$
L=1+z \sum_{k=1}^{Y}(z-1)^{k-1}=\frac{z(z-1)^{Y}-2}{z-2}
$$

for $z \geq 3$ or

$$
L=1+2 Y
$$

for $z=2$ which is the MPS case. The maximal distance between two sites is given by $2 Y$. From eq. (1) and eq. (2) follows a logarithmic scaling of maximal distance with system size $L$ for trees and a linear one for MPSs. Correlation functions in TTNSs with finite bond dimension decay exponentially in function of maximal distance. Hence, in function of system size an algebraic decay is obtained for $z \geq 3$ in contrast to the exponential decay for the MPS $(z=2) .22|25| 26$

Tree tensor networks for quantum chemistry (QC-TTNS) were first studied by Murg et al. $\frac{9 \mid 11}{1}$ for trees with arbitrary coordination number. The complexity of the algorithm as a 
function of the virtual dimension $D$ is given by $\mathcal{O}\left(D^{x+1}\right)$, where $x$ is given by the coordination number of the tensor optimized at each stage. Due to this scaling, Murg et al. restricted themselves to a maximum coordination number of 3 in the network and to a one-site optimization scheme which results in $\mathcal{O}\left(D^{4}\right)$. A two-site optimization scheme in a tree with coordination number of 3 includes optimizing two-site tensors with 4 virtual bonds as can be seen in fig. 1(a). This ultimately results in an expensive $\mathcal{O}\left(D^{5}\right)$.

In DMRG, the usage of a two-site optimization scheme has proved to be advantageous. The two-site scheme is less prone to be stuck in local minima and an automatic redistribution of the virtual dimensions over different symmetry sectors is possible through singular-value decomposition (SVD). ${ }^{15}$ In TTNS it would be opportune to also use two-site optimization. In contrast to DMRG though, two-site optimization for an arbitrary TTNS is accompanied with a heavier polynomial cost than one-site optimization as previously stated. In the work of Nakatani et al.10 this problem is circumvented by introducing half-renormalization. In the half-renormalization step the TTNS is exactly mapped to an MPS. In this MPS, the iterative optimization step is executed at a DMRGlike cost. The mapping of the TTNS to an MPS is still expensive, though.

In this paper, we propose the T3NS ansatz which has considerable advantages compared to a general TTNS. We show that the proposed ansatz enables two-site optimization without any penalty in the polynomial scaling and without the need of mapping through the halfrenormalization scheme.

\subsection{The T3NS ansatz}

Just as in previous works on QC-TTNS, 11 we restrict ourselves to a maximum coordination number $z=3$, to keep calculations feasible. A second restriction we impose, is that only tensors with $z \leq 2$ have physical indices. We call this type of tensors physical tensors. Tensors with $z=3$ are called branching tensors and have exclusively virtual bond indices. An ex- (a)

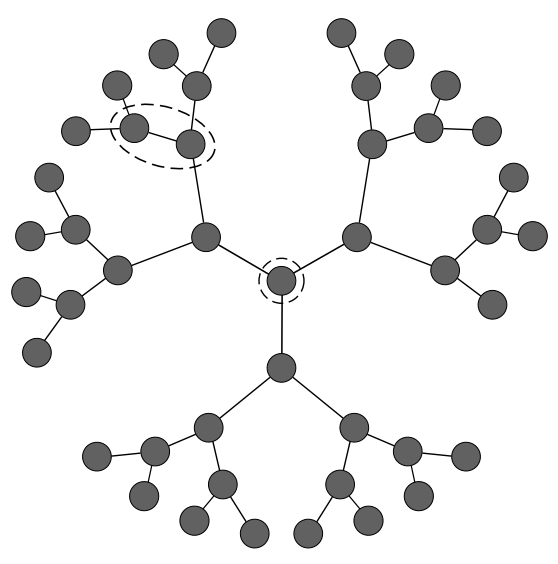

(b)

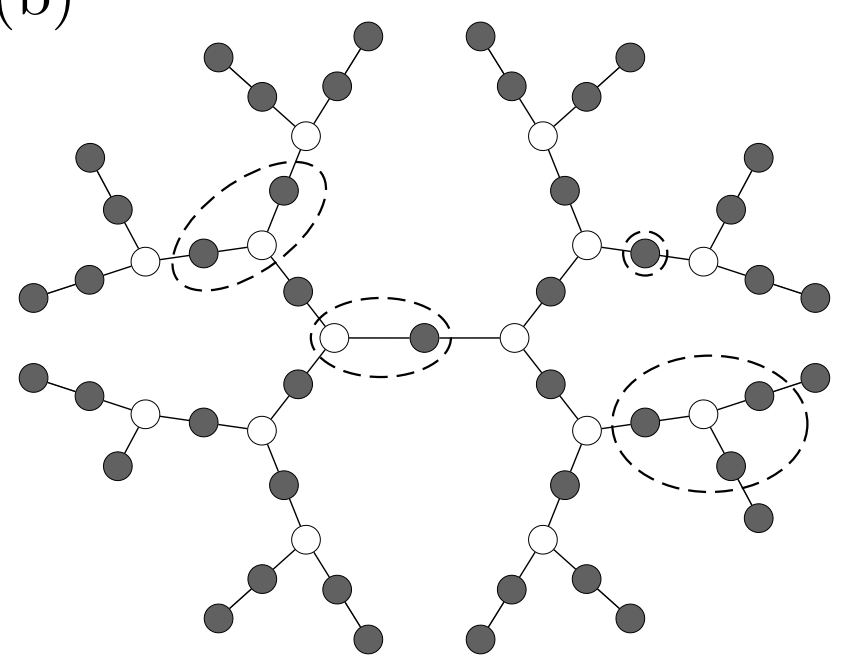

Figure 1: (a) A general TTNS with maximum $z=3$ and 44 orbitals. The tensor optimized during one or two-site optimization is shown by dashed lines, and has maximally 3 or 4 virtual bond indices, respectively. (b) An example of a T3NS with 44 orbitals. The tensor optimized during one, two-, three-, or four-site optimization is shown by the dashed contours, and has maximally 3 virtual bond indices for all cases. Filled circles represent physical tensors and have thus an extra physical index that is not drawn here for simplicity. Empty circles represent branching tensors.

ample of this type of TTNS (the T3NS) is given in fig. 1(b).

The proposed ansatz enables us to go beyond one-site optimization and use two-site, threesite or even four-site optimization with the same polynomial scaling (see fig. 1(b)). Another substantial advantage of T3NS is that every tensor 
has at most three different indices (one physical and two virtual for a physical tensor and three virtual for a branching tensor). Hence no extra substantial difficulties are expected for the implementation of $S U(2)$-symmetry, compared with the MPS formalism. 1922

At this moment our T3NS implementation is able to do two-site, three-site and four-site optimization and it exploits $U(1)$-symmetry. In this paper, only two-site optimization is used. Three- and four-site calculations were executed but the small increase in energy accuracy did not outweigh the extra computational time needed (larger prefactor). However, the ability to do three- and four-site optimization can be useful for orbital optimizations. The usage of $S U(2)$-symmetry will be the subject of subsequent research.

\subsection{Fermionic Networks}

A quantum chemical calculation involves fermions. This introduces extra complexity in the algorithm through the sign change of the wave function when interchanging two fermions. For our implementation we opted for the fermionic network formalism as developed by Bultinck et al. $\stackrel{28}{2}$

In this formalism, fermionic tensors are given by

$$
\left.\left.A=\sum_{\alpha \beta \gamma \delta \ldots} A_{\alpha \beta \gamma \delta \ldots} \mid \alpha\right) \mid \beta\right)(\gamma \mid(\delta \mid \ldots
$$

This is equivalent with the definition for bosonic tensors, but where $\mid \alpha), \mid \beta),(\gamma \mid,(\delta \mid, \ldots$ are instead elements of the so-called super vector space $V$. Bras can be graphically depicted as outgoing tensor legs, while kets are ingoing tensor legs.

The fermionic signs are introduced by the following canonical isomorphism

$$
\begin{aligned}
& \mathcal{F}: V \otimes_{\mathfrak{g}} W \rightarrow W \otimes_{\mathfrak{g}} V \\
&|i\rangle \otimes_{\mathfrak{g}}|j\rangle \rightarrow(-1)^{|i||j|}|j\rangle \otimes_{\mathfrak{g}}|i\rangle,
\end{aligned}
$$

where $V$ and $W$ are super vector spaces and $\otimes_{\mathfrak{g}}$ denotes the graded tensor product. $|i\rangle$ and $|j\rangle$ represent homogeneous basis states. A homoge- neous state is characterized by a definite parity of the state (namely, $|i|,|j| \in\{0,1\}$ ).

For the contraction of fermionic tensors, a second mapping is introduced:

$$
\mathcal{C}: V^{*} \otimes_{\mathfrak{g}} V \rightarrow \mathbb{C}:\left\langle\psi\left|\otimes_{\mathfrak{g}}\right| \phi\right\rangle \rightarrow\langle\psi \mid \phi\rangle,
$$

where $V$ and $V^{*}$ are the super vector space and its dual space, respectively.

When contracting two fermionic tensors, the states of the tensors should first be ordered appropriately through successive usage of eq. (4) before using eq. (5). No explicit ordering of the orbitals is needed this way, but this is implicitly fixed by the initial order of the indices in the different tensors of the network. For further details we refer to ref. 28 .

\subsection{Resource requirements of the algorithm}

In this section the computational complexity and the memory requirements of the algorithm are discussed. For the implementation of the algorithm, we opted for the usage of (complementary) renormalized operators, just as in previous works on QC-TTNS.9-11 This approach for the efficient calculation of expectation values has also been heavily used in highly optimized QC-DMRG.

Another technique, the so-called Matrix Product Operator (MPO) formalism, has also been formulated for the quantum chemistry Hamiltonian. In this formalism, the Hamiltonian is represented by a tensor network too, consisting of different MPOs (as opposed to MPSs for the wave function). To obtain an efficient MPO representation of the Hamiltonian, the bond dimension of the MPO should be at least of the same order as the number of renormalized operators used, i.e. $\mathcal{O}\left(k^{2}\right)$, with $k$ the number of orbitals. When such a representation is found, one still has to exploit the extra sparsity of the MPOs to obtain the same cost as with renormalized operators. For QC-DMRG, several methods of obtaining such MPO and exploiting the extra sparsity are already known. 23124

Equivalently for QC-TTNS, the QC- 
Hamiltonian can be formulated in the Tensor Network Operator (TNO) language. While the Hamiltonian is represented by a linear tensor network in the MPO formalism, the Hamiltonian can also be represented by a tree tensor network through usage of TNO's. Again, a first condition for an efficient TNO is a scaling of $\mathcal{O}\left(k^{2}\right)$ for the bond dimension. Just as in DMRG exploitation of sparsity is needed to obtain similar costs as with renormalized operators. Failing to do so is even more catastrophic for QC-TTNS than for QC-DMRG. The methods proposed in refs. 23,24 are not readily translatable to QC-TTNS or do not produce the same scaling as with renormalized operators. Because of this, we opted for the usage of renormalized operators.

Table 1: Resource requirements of DMRG and T3NS with renormalized operators. The underlined terms correspond with the complexity of the most intensive part of the algorithm, i.e. the matrix-vector product used in the iterative solver.

\begin{tabular}{rc|c} 
& DMRG & T3NS \\
\cline { 2 - 3 } CPU time: & $\mathcal{O}\left(k^{4} D^{2}+k^{3} D^{3}\right)$ & $\mathcal{O}\left(k^{5} D^{2}+k^{3} D^{4}\right)$ \\
Memory: & $\mathcal{O}\left(k^{2} D^{2}\right)$ & $\mathcal{O}\left(k^{2} D^{2}+k D^{3}\right)$ \\
Disk: & $\mathcal{O}\left(k^{3} D^{2}\right)$ & $\mathcal{O}\left(k^{3} D^{2}+k D^{3}\right)$
\end{tabular}

The predicted scaling of CPU time, memory usage, and disk usage are given in Table 1 and compared with DMRG. The most time consuming part of the algorithm is the iteratively executed matrix-vector product of the effective Hamiltonian with the two-site tensor $\left(H_{\text {eff }} \Psi\right)$. Due to the usage of complementary renormalized operators, the effective Hamiltonian is constructed out of $\mathcal{O}\left(k^{2}\right)$ different terms, for both DMRG and T3NS. However, the cost of constructing each term scales as $\mathcal{O}\left(D^{4}\right)$ for T3NS instead of $\mathcal{O}\left(D^{3}\right)$ for DMRG.

The other leading term in the CPU time per sweep is due to updating the renormalized operators. The most intensive type of update for renormalized operators is when two sets of renormalized operators have to be recombined in a new set, this by using a branching tensor. The most intensive type of recombination oc- curs when a single operator in both sets has to be updated to a complimentary double operator. This results in $\mathcal{O}\left(k^{5} D^{2}+k^{3} D^{4}\right)$ per sweep, as can be seen in fig. 2 .

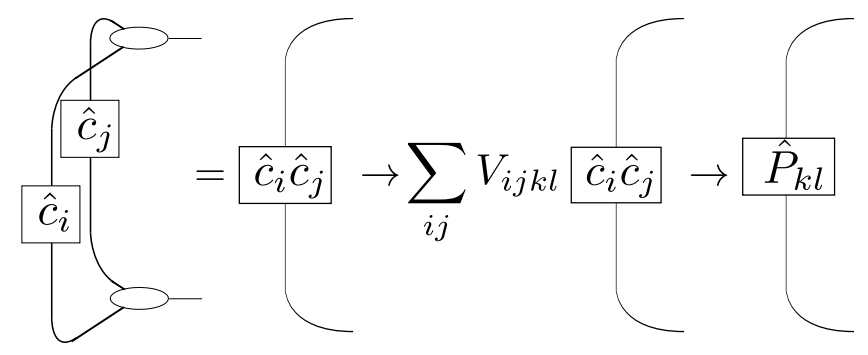

Figure 2: A graphical depiction of the most intensive part of updating the renormalized operators, i.e. the recombination of two single operators into a double complementary operator. First, the single operators are combined to a double operator with the aid of a branching tensor $\left(\mathcal{O}\left(k^{2} D^{4}\right)\right)$. In the second stage, the newly formed double operators are summed together with their potential terms into the different double complementary operators $\left(\mathcal{O}\left(k^{4} D^{2}\right)\right)$. Since there are $\mathcal{O}(k)$ occasions per sweep for this, we obtain $\mathcal{O}\left(k^{5} D^{2}+k^{3} D^{4}\right)$.

At a fixed system size $k$ and bond dimension $D$, the speed of a sweep is still dependent of the particular shape of the tree. While one can only make one shape of MPS-chain for a fixed system size, this is not true for trees.

\subsection{Shape of the tree, orbital or- dering and choice}

As previously stated, the different shapes for the tree at fixed $k$ introduce additional freedom that is not present in DMRG. It is clear that the particular shape will influence the speed and the accuracy of the calculations. The orbital ordering in the network is also of importance for the accuracy of the calculations. This freedom is also present in DMRG and multiple methods for ordering the orbitals exist (e.g. through use of the mutual information ${ }^{11}$ or the exchange integral ${ }^{10}$ have been studied). Similar methods can be used to optimize the shape of the tree.

Finally, the orbital choice and orbital optimization is also of importance for TTNS and DMRG calculations. Quite some research has 
been done for this in DMRG. $\stackrel{29}{24}$ In TTNS, orbital optimization by canonical transformations has been studied and used. $\underline{9}$

In this paper, we group orbitals belonging to the same spatial irrep as much as possible and connect the irreps in the center of the tree. The used trees and orbital orderings are given in the supplementary material. Within one irrep, the orbitals are ordered such that the orbitals closest to the Fermi level (for $\mathrm{LiF}$ and $\mathrm{N}_{2}$ ) or the orbitals with highest single-orbital entropy (for $\left[\mathrm{Cu}_{2} \mathrm{O}_{2}\right]^{2+}$ ) are closest to the center of the tree. After experimenting with a few different orderings of the orbitals, this proved to be the most successful one. Another degree of freedom is the choice of the orbitals. In this paper, we only use Hartree-Fock orbitals. No orbital optimization is executed, as this paper serves only as an initial description of our particular T3NS ansatz.

Optimization of the shape of the network, the orbital order, or the orbitals themselves will be the subject of subsequent research.

\section{$3 \quad$ Numerical results}

In this section, we compare the T3NS ansatz with the MPS ansatz. Energy errors and CPU times are compared in function of the bond dimension. We study $\mathrm{LiF}$ and $\mathrm{N}_{2}$ at their equilibrium bond length $(r=3.05$ a.u. and $r=2.118$ a.u., respectively). For $\mathrm{LiF}$ we also calculations at $r=12$ a.u. and $r=13.7$ a.u. $\mathrm{LiF}$ and $\mathrm{N}_{2}$ are two systems that don't particularly call for a tree-shaped topology representation. However, as we will show, a similar accuracy is already obtained with the T3NS at considerable lower bond dimension as compared with DMRG for both systems. This fact gives us hope that the more complex entanglement topology will prove even more its merits in larger molecules, since the orbitals can be easier arranged in groups of highly entangled orbitals. 18

$\mathrm{LiF}$ and $\mathrm{N}_{2}$ are studied for different bond dimensions by T3NS with $U(1)$-symmetry and DMRG with $U(1)$-symmetry. LiF is also studied with DMRG with $S U(2)$-symmetry. For both T3NS-U(1) and DMRG- $U(1)$ we use our own implementation of the T3NS ansatz. It is of course also possible to do DMRG- $U(1)$ since this is just a subset of the possible treeshaped geometries. For DMRG-SU(2) we use the CheMPS2 software program developed by S. Wouters. 21/22/35/36

Both systems are popular benchmarks for methods and their ability to take strong electron correlations into account, and both systems have been studied in previous papers about QC-TTNS (LiF in ref. 11 and $\mathrm{N}_{2}$ in ref. 10).

To test the T3NS ansatz in larger systems, we perform calculations on the bis $(\mu$-oxo $)$ and the $\mu-\eta^{2}: \eta^{2}$ peroxo $\left[\mathrm{Cu}_{2} \mathrm{O}_{2}\right]^{2+}$ isomers. We compare the energy gaps between the two isomers obtained by T3NS with previously published values. 33137 We We also compare our complete active space (CAS) ground-state energies with other calculations executed in the same active space. This way we keep comparisons fair.

\subsection{Results for $\mathrm{LiF}$}

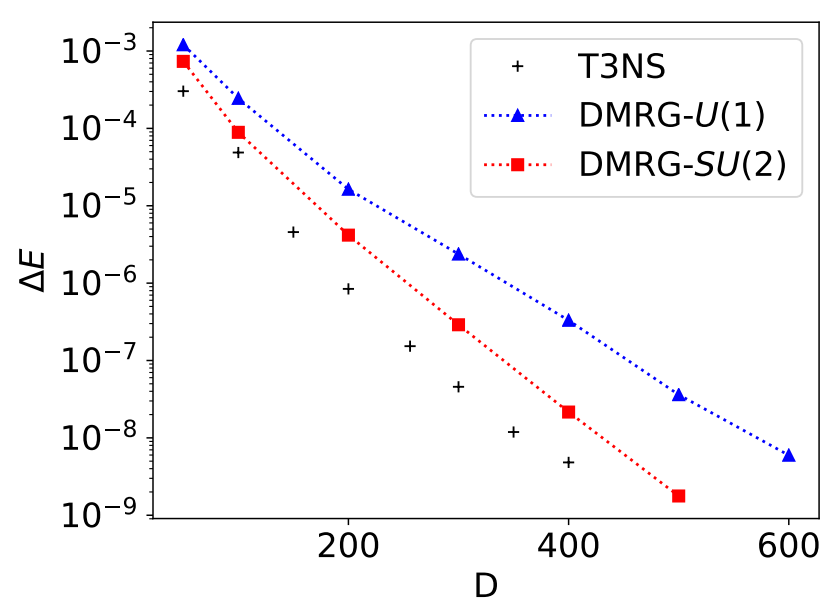

Figure 3: Energy difference of DMRG and T3NS calculations with respect to the FCI energy for $\mathrm{LiF}$ at equilibrium bond length $r=$ 3.05 a.u. FCI energies are obtained from ref. 11. The calculations are done at different (reduced) bond dimensions. The TTNS geometry is given in the supplementary material.

The first system we study with the new T3NS ansatz is LiF. We perform calculations at equilibrium bond length ( $r=3.05$ a.u.), at $r=12$ 


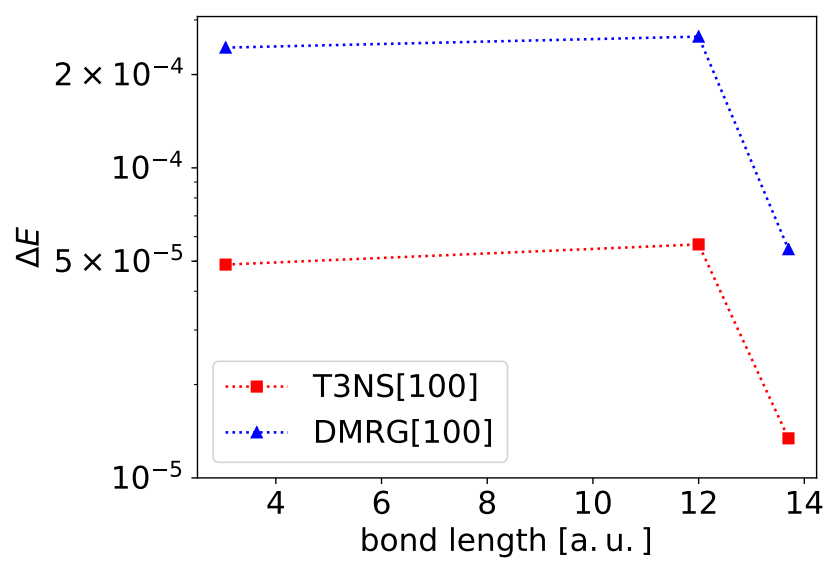

Figure 4: Energy difference of DMRG- $U(1)$ and T3NS- $U(1)$ calculations with respect to the FCI energy for LiF at bond length $r=3.05,12$ and 13.7 a.u. FCI energies are obtained from ref. 11. The calculations are done at $D=100$ for both DMRG and T3NS. The TTNS geometry is given in the supplementary material.

Table 2: Some timings for T3NS and DMRG calculations of $\mathrm{LiF}$ at equilibrium bond length. Used bond dimensions are given in square brackets. Both T3NS and DMRG are executed with our own implementation to keep comparison fair.

\begin{tabular}{l|c|c} 
& CPU time last sweep & total CPU time \\
\hline T3NS[100] & $96 \mathrm{sec}$ & $428 \mathrm{sec}$ \\
T3NS[400] & $1000 \mathrm{sec}$ & $2240 \mathrm{sec}$ \\
\hline DMRG[100] & $48 \mathrm{sec}$ & $600 \mathrm{sec}$ \\
DMRG[600] & $640 \mathrm{sec}$ & $1884 \mathrm{sec}$
\end{tabular}

a.u. where an avoided crossing occurs, and at large bond length ( $r=13.7$ a.u.). The bond lengths are expressed in atomic units. Calculations are performed in a CAS of size (6e, 25). The atomic orbital basis from Bauschlicher and Langhoff ${ }^{45}$ was used. For the active space calculations, the $1 \sigma, 2 \sigma$ and $3 \sigma$ orbitals were kept frozen. The same basis set and active space is used in ref. 11. Ground state energies were calculated by using T3NS- $U(1)$, DMRG- $U(1)$ and DMRG-SU(2) with several bond dimensions. In the case of DMRG-SU(2), the quoted bond dimension is the reduced one, where the additional $S U(2)$-symmetry is taken into account.21 FCI energies were easily recovered through the T3NS. Accuracies in the order of $10^{-8} \mathrm{E}_{\mathrm{h}}$ were obtained for all bond lengths at $D=400$. The accuracy of DMRG and T3NS in relation to the bond dimension is given in fig. 3 for $\mathrm{LiF}$ in equilibrium. As expected, a lower bond dimension is needed for T3NS for a similar accuracy as in DMRG. In fig. 4, the accuracy of DMRG and T3NS is given for different bond lengths at a low bond dimension $(D=100)$.

Lastly, some wall times for the T3NS and DMRG calculations are given in table 2 for $r=3.05$. At $D=100$, a sweep is twice as slow in T3NS as in DMRG, as can be expected. However, less sweeps are needed until convergence which ultimately results in a faster calculation with higher accuracy. The need for fewer sweeps in T3NS is something we noticed quite consistently. For DMRG at $D=600$ and T3NS at $D=400$ both accuracy and total wall time are comparable. We would like to note that these remarks on timing are by no means conclusive since the speed and accuracy of both T3NS and DMRG are heavily dependent on orbital ordering and initial guess. In these calculations, a random initial guess and a rather intuitive orbital ordering was used. These remarks are merely to illustrate the competitiveness of our T3NS ansatz with DMRG.

The used TTNS geometries and orbital orderings are given in the supplementary material.

\subsection{Results for $\mathrm{N}_{2}$}

Table 3: Some CPU times for T3NS and DMRG calculations of $\mathrm{N}_{2}$ at equilibrium bond length. Both T3NS and DMRG are executed with our own implementation to keep comparison fair. Ordering of the orbitals on the network are given in the supplementary material.

\begin{tabular}{l|c|c} 
& CPU time last sweep & total CPU time \\
\hline T3NS[100] & $560 \mathrm{sec}$ & $1440 \mathrm{sec}$ \\
T3NS[300] & $4 \mathrm{~h}$ & $17 \mathrm{~h}$ \\
T3NS[500] & $16 \mathrm{~h}$ & $96 \mathrm{~h}$ \\
T3NS[700] & $66 \mathrm{~h}$ & $237 \mathrm{~h}$ \\
\hline DMRG[100] & $160 \mathrm{sec}$ & $3800 \mathrm{sec}$ \\
DMRG[500] & $2050 \mathrm{sec}$ & $9 \mathrm{~h}$ \\
DMRG[1000] & $2 \mathrm{~h}$ & $27 \mathrm{~h}$
\end{tabular}

The second benchmark system for our T3NS ansatz is the nitrogen dimer at equilibrium 


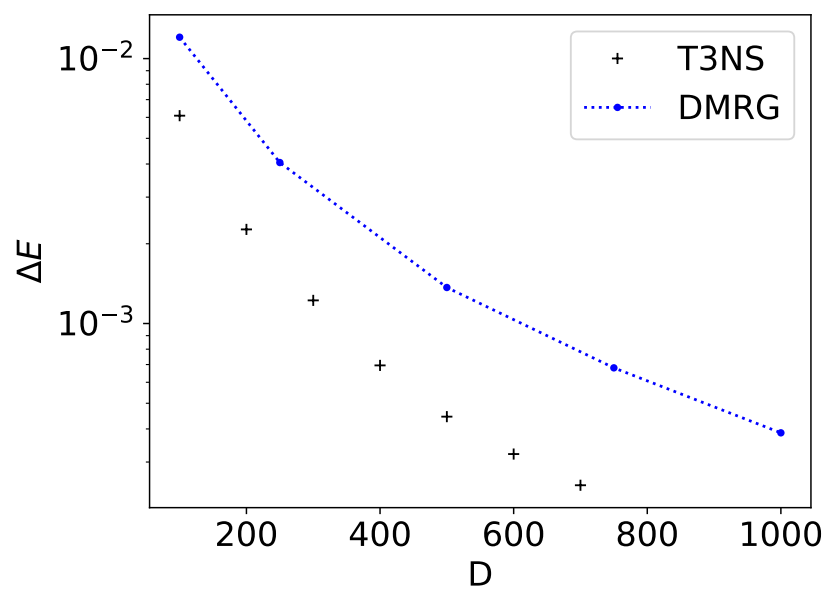

Figure 5: $\quad$ Energy difference of DMRG and T3NS calculations for $\mathrm{N}_{2}$ with respect to CCSDTQPH energy (-109.282172 $\left.\mathrm{E}_{\mathrm{h}}\right) . \underline{46}$ The calculations are done at different bond dimensions. The TTNS geometry is given in the supplementary material.

(bond length: 2.118 a.u.). This is a popular molecule for benchmarking methods in their ability to describe strong electron correlation accurately. Because of this it has also been discussed by Nakatani et al. in their TTNS paper. ${ }^{[0]}$ They studied the nitrogen dimer in a ccpVDZ basis set in a frozen core active space (10e, 26), keeping the 1s electrons of nitrogen fixed. DMRG ${ }^{46}$ and $\mathrm{FCI}^{47}$ calculations have also been previously executed for this active space. In this paper we execute all-electron calculations $(14 \mathrm{e}, 28)$ for the nitrogen dimer in a cc-pVDZ basis set and compare them with the most accurate results obtained in ref. 46 through coupled cluster on the SDTQPH level.

Several calculations have been executed at different bond dimensions for T3NS and DMRG with $U(1) \times U(1)$-symmetry. The obtained energy differences with respect to CCSDTQPH are given in fig. 5 for bond dimensions up to 1000 for DMRG and up to 700 for T3NS. Comparable energies are obtained for T3NS at half the bond dimension needed for DMRG. This is consistent with the conclusion from the frozen core TTNS calculations in ref. 10.

CPU times are given in table 3 for T3NS$U(1)$ and DMRG- $U(1)$ calculations. Similar conclusions can be made in comparison with
LiF. For $D=100$, T3NS-sweeps take longer than DMRG-sweeps, but the number of sweeps needed for convergence from a random initial guess is considerably lower. This ultimately results in a lower wall time. At $D=1000$ for DMRG and $D=500$ for T3NS, obtained accuracies are comparable. Wall times for T3NS are higher though than for DMRG, but still in the same order.

\subsection{Results for the bisoxo and peroxo isomer of $\left[\mathrm{Cu}_{2} \mathrm{O}_{2}\right]^{2+}$}

As a last benchmark system, we study the bisoxo $(\mu$-oxo $)$ and peroxo isomers of $\left[\mathrm{Cu}_{2} \mathrm{O}_{2}\right]^{2+}$, and in particular their energy gap. These transition metal clusters have been studied with a wide range of ab initio methods like CASSCF and CASPT2 (complete active space self consistent field theory with perturbation theory up to second order) ${ }^{37}$ and RASPT2 (restricted active space self consistent field theory with perturbation theory up to second order). $\stackrel{38}{+}$ However, the small active spaces used for CASPT2 and RASPT2 showed to be insufficient. Later on, the usage of DMRG-based methods allowed to take a considerably larger active space into account, yielding improved results. 3313944

In this paper, we use the T3NS algorithm to treat the two isomers in a $(26 \mathrm{e}, 44)$ active space. We use the same active space and basis set as in ref. 39 and 33. Results are given in table 4 . Energies of the isomers in the used active spaces are very comparable to the ones in ref. 33. Furthermore, the energy gap between the two isomers are in the same region as previously executed DMRG calculations.

These results are especially promising since no advanced methods were used to augment the T3NS calculations in contrast to the previous research with DMRG for this system. At this moment our algorithm starts from a random initial guess, no effort was made in avoiding local minima and an intuitive orbital ordering was used. In contrast, previous DMRG research included the configuration interaction based dynamically extended active space (CI-DEAS) procedure $^{33}$ or adding perturbative noise to the tensors ${ }^{44}$ to avoid local minima. Orbitals 
Table 4: Energy gaps between the bis $\left(\mu\right.$-oxo) and $\mu-\eta^{2}: \eta^{2}$ peroxo $\left[\mathrm{Cu}_{2} \mathrm{O}_{2}\right]^{2+}$ isomers from T3NS calculations of this paper and previous calculations. The energy gaps are given in $\mathrm{kcal} / \mathrm{mol}$. Ground state energies are given for the T3NS calculations and DMRG calculations from previous research using the same active space and are given in Hartree. Bond dimensions used for the T3NS and DMRG calculations are given in square brackets.

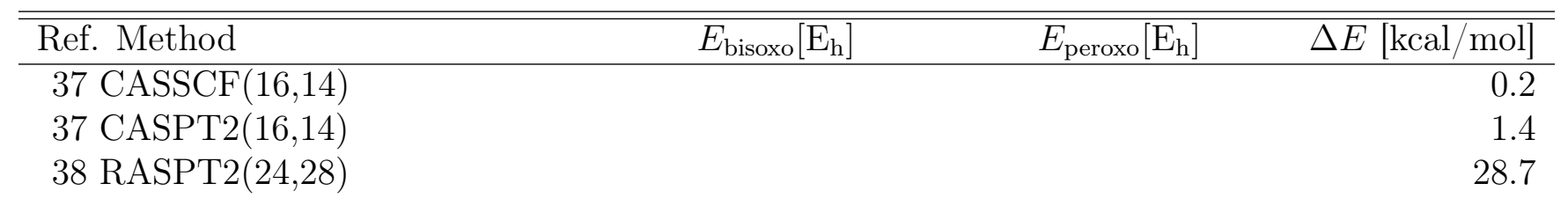

40 DMRG(32,62)[2400]

Some previously published DMRG energies

41] DMRG(28,32)[2048]-SCF/CT

35.6

43 DMRG $(32,28)[4000]$

27.0

44] DMRG $(24,24)[1500]-\mathrm{SCF}^{*}$

21.8

44 DMRG $(24,24)[1500]-C A S P T 2 *$

35.1

39 DMRG $(26,44)[800]$

42 DMRG $(26,44)[128]$

33 DMRG $(26,44)\left[256 / 1024 / 10^{-5}\right]^{\dagger}$

$-541.46779$

$-541.47308$

$-541.53853$

23.2

18.5

26.1

$-541.51470$

26.7

T3NS $(26,44)[50]$

T3NS $(26,44)[100]$

T3NS $(26,44)[200]$

T3NS $(26,44)[300]$

T3NS $(26,44)[500]$
T3NS calculations

$-541.48773$

$-541.52352$

$-541.53284$

$-541.53556$

$-541.53820$
$-541.56999$

$-541.57166$

$-541.57717$

$-541.57966$

$-541.58094$
51.6

30.2

27.8

27.7

26.8

*: Bond dimensions given for these calculations are reduced bond dimensions.

$\dagger$ : This calculation uses the DBSS method and CI-DEAS as initialization procedure. The square brackets state that a minimum of $D=256$ is used at every bond, the CI-DEAS procedure starts with $D=1024$ and a maximum discarded weight of $10^{-5}$ is aimed for. Maximum bond dimensions around 2000 were reported for both clusters during these calculations. $\frac{33}{33}$

were ordered by minimizing quantum entanglement using the Fiedler vector, ${ }^{33 / 44}$ or a genetic algorithm. ${ }^{44}$ Other methods used to augment the results were DMRG-SCF (self consistent field), ${ }_{11}$ DMRG-SCF with canonical transformation theory (DMRG-SCF/CT) ${ }^{41}$ or DMRGCASPT2 to take dynamical correlation into account. In ref. 33 dynamic block state selection (DBSS) was used to tune the bond dimension. Instead of a fixed bond dimension, a maximum discarded weight is used. In this way, the bond dimension at every bond is tailored to stay below this maximum discarded weight. DBSS is easily implementable once two-site optimization is used, like in our T3NS algorithm.
Since we noted that the discarded weight was dominant in very few bonds while it was orders lower in other bonds, we think that DBSS can also yield a substantial improvement in the T3NS algorithm.

To check if we got stuck in local minima, the ground state wave function of the bisoxo isomer obtained through T3NS[500] was compressed to a lower bond dimension. The compressed wave function was then reoptimized at this lower bond dimension and we found a ground state energy of $-541.50527,-541.52387$ and -541.53327 Hartree for $D=50,100$ and 200, respectively. Comparison with the results obtained through random initialization in ta- 
ble 4 makes the problem of local minima quite clear and shows us that preventing local minima can improve our results significantly, especially at low bond dimension.

\section{Conclusion}

In this paper, we have presented a new variational ansatz, the T3NS ansatz. This is a subclass of the general TTNS ansatz which has considerable advantages. By interspersing physical and branching tensors in the network, two-site optimization (and even three- and four-site optimization) becomes feasible at the same polynomial cost as one-site optimization. Furthermore, both physical and branching tensors in the T3NS network have at most 3 indices which allows a simple implementation of symmetries. In this way, we join the computational efficiency of the MPS with the richer entanglement description of the TTNS.

As a proof-of-concept, calculations were executed for $\mathrm{LiF}, \mathrm{N}_{2}$ and $\left[\mathrm{Cu}_{2} \mathrm{O}_{2}\right]^{2+}$ with our T3NS implementation. Accuracies and timings were compared with DMRG. Similar accuracies at lower bond dimensions were obtained with T3NS. For $\left[\mathrm{Cu}_{2} \mathrm{O}_{2}\right]^{2+}$ in a $(26 \mathrm{e}, 44)$ active space, a comparable accuracy was obtained at $D=500$ for T3NS and previously published DMRG with DBSS and a maximal bond dimension of around 2000. $\underline{33}$

For our proof-of-concept, no great effort was made in optimizing the orbital ordering or avoiding local minima. Furthermore, only the $U(1)$-symmetry of the QCHamiltonian was exploited, but no point group and $S U(2)$-symmetry. One could also consider post-T3NS methods in close similarity to post-DMRG methods. Some examples are DMRG-SCF, ${ }^{32}$ DMRG-CASPT2 $2^{48}$ and DMRG-TCCSD (DMRG-tailored coupled cluster with single and double excitations). ${ }^{49}$ These topics will be of interest in future research.

Acknowledgement K.G. acknowledges support from the Research Foundation Flanders (FWO Vlaanderen). Ö.L. acknowledges support from the Hungarian National Research, Development and Innovation Office (NKFIH) through Grant No. K120569 and the Hungarian Quantum Technology National Excellence Program (Project No. 2017-1.2.1-NKP-201700001).

\section{Supporting Information Avail- able}

The following files are available free of charge.

The following supporting files are available.

- trees.pdf: The used tree tensor networks for the $\mathrm{LiF}, \mathrm{N}_{2}$ and $\left[\mathrm{Cu}_{2} \mathrm{O}_{2}\right]^{2+}$ calculations.

\section{References}

(1) White, S. R. Density matrix formulation for quantum renormalization groups. Phys. Rev. Lett. 1992, 69, 2863-2866.

(2) White, S. R. Density-matrix algorithms for quantum renormalization groups. Phys. Rev. B 1993, 48, 10345-10356.

(3) Xiang, T. Density-matrix renormalization-group method in momentum space. Phys. Rev. B 1996, 53, R10445-R10448.

(4) White, S. R.; Martin, R. L. Ab initio quantum chemistry using the density matrix renormalization group. Journal of Chemical Physics 1999, 110, 4127-4130.

(5) Östlund, S.; Rommer, S. Thermodynamic Limit of Density Matrix Renormalization. Phys. Rev. Lett. 1995, 75, 3537-3540.

(6) Rommer, S.; Östlund, S. Class of ansatz wave functions for one-dimensional spin systems and their relation to the density matrix renormalization group. Phys. Rev. B 1997, 55, 2164-2181.

(7) Verstraete, F.; Cirac, J. I. Renormalization algorithms for Quantum-Many Body Systems in two and higher dimensions. eprint arXiv:cond-mat/0407066 2004, 
(8) Vidal, G. Entanglement Renormalization. Phys. Rev. Lett. 2007, 99, 220405.

(9) Murg, V.; Verstraete, F.; Legeza, Ö.; Noack, R. M. Simulating strongly correlated quantum systems with tree tensor networks. Phys. Rev. B 2010, 82, 205105.

(10) Nakatani, N.; Chan, G. K.-L. Efficient tree tensor network states (TTNS) for quantum chemistry: Generalizations of the density matrix renormalization group algorithm. Journal of Chemical Physics 2013, 138, 134113-134113.

(11) Murg, V.; Verstraete, F.; Schneider, R.; Nagy, P.; Legeza, Ö. Tree tensor network state with variable tensor order: an efficient multireference method for strongly correlated systems. Journal of Chemical Theory and Computation 2015, 11, 10271036 .

(12) Marti, K. H.; Bauer, B.; Reiher, M.; Troyer, M.; Verstraete, F. Complete-graph tensor network states: a new fermionic wave function ansatz for molecules. New Journal of Physics 2010, 12, 103008.

(13) Kovyrshin, A.; Reiher, M. Self-adaptive tensor network states with multi-site correlators. The Journal of Chemical Physics 2017, 147, 214111.

(14) Chan, G. K.-L.; Head-Gordon, M. Highly correlated calculations with a polynomial cost algorithm: A study of the density matrix renormalization group. The Journal of Chemical Physics 2002, 116, 4462-4476.

(15) Legeza, Ö.; Röder, J.; Hess, B. A. Controlling the accuracy of the density-matrix renormalization-group method: The dynamical block state selection approach. Phys. Rev. B 2003, 67, 125114.

(16) Schollwöck, U. The density-matrix renormalization group in the age of matrix product states. Annals of Physics 2011, 326, 96-192.
(17) Orús, R. Advances on tensor network theory: symmetries, fermions, entanglement, and holography. European Physical Journal B 2014, 87, 280.

(18) Szalay, S.; Pfeffer, M.; Murg, V.; Barcza, G.; Verstraete, F.; Schneider, R.; Legeza, Ö. Tensor product methods and entanglement optimization for ab initio quantum chemistry. International Journal of Quantum Chemistry 2015, 115, 13421391.

(19) McCulloch, I. P.; Gulácsi, M. The non-Abelian density matrix renormalization group algorithm. Europhysics Letters 2002, 57, 852-858.

(20) Sharma, S.; Chan, G. K.-L. Spin-adapted density matrix renormalization group algorithms for quantum chemistry. Journal of Chemical Physics 2012, 136, 124121124121.

(21) Wouters, S.; Poelmans, W.; Ayers, P. W.; Neck, D. V. CheMPS2: A free open-source spin-adapted implementation of the density matrix renormalization group for ab initio quantum chemistry. Computer Physics Communications 2014, 185, 1501 -1514 .

(22) Wouters, S.; Van Neck, D. The density matrix renormalization group for ab initio quantum chemistry. The European Physical Journal D 2014, 68, 272.

(23) Keller, S.; Dolfi, M.; Troyer, M.; Reiher, M. An efficient matrix product operator representation of the quantum chemical Hamiltonian. Journal of Chemical Physics 2015, 143, 244118.

(24) Chan, G. K.-L.; Keselman, A.; Nakatani, N.; Li, Z.; White, S. R. Matrix product operators, matrix product states, and ab initio density matrix renormalization group algorithms. The Journal of Chemical Physics 2016, 145, 014102. 
(25) Shi, Y.-Y.; Duan, L.-M.; Vidal, G. Classical simulation of quantum many-body systems with a tree tensor network. Phys. Rev. A 2006, 74, 022320.

(26) Ferris, A. J. Area law and real-space renormalization. Phys. Rev. B 2013, 87, 125139 .

(27) Tóth, A. I.; Moca, C. P.; Legeza, Ö.; Zaránd, G. Density matrix numerical renormalization group for non-Abelian symmetries. Phys. Rev. $B$ 2008, 78, 245109.

(28) Bultinck, N.; Williamson, D. J.; Haegeman, J.; Verstraete, F. Fermionic matrix product states and one-dimensional topological phases. Phys. Rev. B 2017, 95, 075108.

(29) Legeza, Ö.; Sólyom, J. Optimizing the density-matrix renormalization group method using quantum information entropy. Phys. Rev. B 2003, 68, 195116.

(30) Rissler, J.; Noack, R. M.; White, S. R. Measuring orbital interaction using quantum information theory. Chemical Physics 2006, 323, 519-531.

(31) Ghosh, D.; Hachmann, J.; Yanai, T.; Chan, G. K.-L. Orbital optimization in the density matrix renormalization group, with applications to polyenes and $\beta$ carotene. Journal of Chemical Physics 2008, 128, 144117-144117.

(32) Zgid, D.; Nooijen, M. The density matrix renormalization group self-consistent field method: Orbital optimization with the density matrix renormalization group method in the active space. The Journal of Chemical Physics 2008, 128, 144116.

(33) Barcza, G.; Legeza, Ö.; Marti, K. H.; Reiher, M. Quantum-information analysis of electronic states of different molecular structures. Phys. Rev. A 2011, 83, 012508.
(34) Krumnow, C.; Veis, L.; Legeza, Ö.; Eisert, J. Fermionic Orbital Optimization in Tensor Network States. Phys. Rev. Lett. 2016, 11\%, 210402.

(35) Wouters, S.; Bogaerts, T.; Van Der Voort, P.; Van Speybroeck, V.; Van Neck, D. Communication: DMRG-SCF study of the singlet, triplet, and quintet states of oxo-Mn(Salen). Journal of Chemical Physics 2014, 140, 241103.

(36) Wouters, S.; Van Speybroeck, V.; Van Neck, D. DMRG-CASPT2 study of the longitudinal static second hyperpolarizability of all-trans polyenes. Journal of Chemical Physics 2016, 145, 054120.

(37) Cramer, C. J.; Włoch, M.; Piecuch, P.; Puzzarini, C.; Gagliardi, L. Theoretical Models on the Cu2O2 Torture Track: Mechanistic Implications for Oxytyrosinase and Small-Molecule Analogues. The Journal of Physical Chemistry A 2006, 110, 1991-2004, PMID: 16451035.

(38) Malmqvist, P. A.; Pierloot, K.; Shahi, A. R. M.; Cramer, C. J.; Gagliardi, L. The restricted active space followed by second-order perturbation theory method: Theory and application to the study of $\mathrm{CuO} 2$ and $\mathrm{Cu} 2 \mathrm{O} 2$ systems. The Journal of Chemical Physics 2008, 128, 204109.

(39) Marti, K. H.; Ondík, I. M.; Moritz, G.; Reiher, M. Density matrix renormalization group calculations on relative energies of transition metal complexes and clusters. The Journal of Chemical Physics 2008, 128, 014104.

(40) Kurashige, Y.; Yanai, T. Highperformance ab initio density matrix renormalization group method: Applicability to large-scale multireference problems for metal compounds. The Journal of Chemical Physics 2009, 130, 234114.

(41) Yanai, T.; Kurashige, Y.; Neuscamman, E.; Chan, G. K.-L. Multireference quantum chemistry through a joint 
density matrix renormalization group and canonical transformation theory. The Journal of Chemical Physics 2010, 132, 024105 .

(42) Marti, K. H.; Reiher, M. The Density Matrix Renormalization Group Algorithm in Quantum Chemistry. Zeitschrift für Physikalische Chemie 2010, 224, 583599.

(43) Stein, C. J.; Reiher, M. Automated Selection of Active Orbital Spaces. Journal of Chemical Theory and Computation 2016, 12, 1760-1771, PMID: 26959891.

(44) Phung, Q. M.; Wouters, S.; Pierloot, K. Cumulant Approximated Second-Order Perturbation Theory Based on the Density Matrix Renormalization Group for Transition Metal Complexes: A Benchmark Study. Journal of Chemical Theory and Computation 2016, 12, 4352-4361, PMID: 27547847.

(45) Bauschlicher, C. W.; Langhoff, S. R. Full configuration-interaction study of the ionic-neutral curve crossing in $\mathrm{LiF}$. The Journal of Chemical Physics 1988, 89, 4246-4254.

(46) Chan, G. K.-L.; Kállay, M.; Gauss, J. State-of-the-art density matrix renormalization group and coupled cluster theory studies of the nitrogen binding curve. The Journal of Chemical Physics 2004, 121, 6110-6116.

(47) Larsen, H.; Olsen, J.; Jørgensen, P.; Christiansen, O. Full configuration interaction benchmarking of coupled-cluster models for the lowest singlet energy surfaces of N2. The Journal of Chemical Physics 2000, 113, 6677-6686.

(48) Kurashige, Y.; Yanai, T. Second-order perturbation theory with a density matrix renormalization group self-consistent field reference function: Theory and application to the study of chromium dimer. The Journal of Chemical Physics 2011, 135, 094104.
(49) Veis, L.; Antalík, A.; Brabec, J.; Neese, F.; Legeza, Ö.; Pittner, J. Coupled Cluster Method with Single and Double Excitations Tailored by Matrix Product State Wave Functions. The Journal of Physical Chemistry Letters 2016, 7, 4072-4078. 


\section{Supporting information for: \\ T3NS: three-legged tree tensor network states}
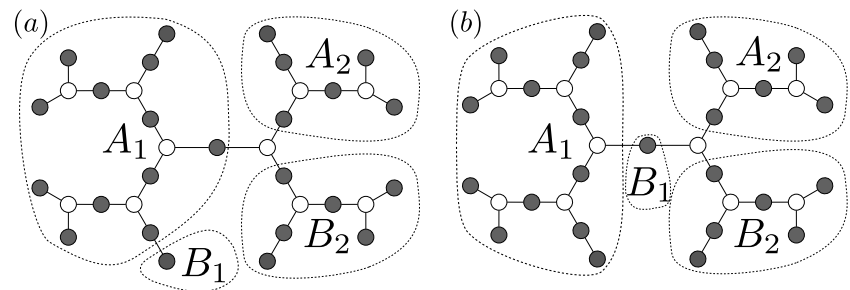

Figure S1: Tree-shaped network for $\mathrm{LiF}$ in the $(6 \mathrm{e}, 25)$ active space. The orbitals belonging to the same irreducible representation are grouped. LiF belongs to the $C_{2 v}$ point group. $A_{1}, A_{2}, B_{1}$ and $B_{2}$ are the Mulliken symbols of the irreducible representations of $C_{2 v}$. The orbitals closest to the Fermi level are put as close to the center as possible.

(a) is used for the equilibrium bond length $r=3.05$ a.u. and (b) is used for the two calculations at large separation $(r=12$ a.u. and $r=13.7$ a.u.).

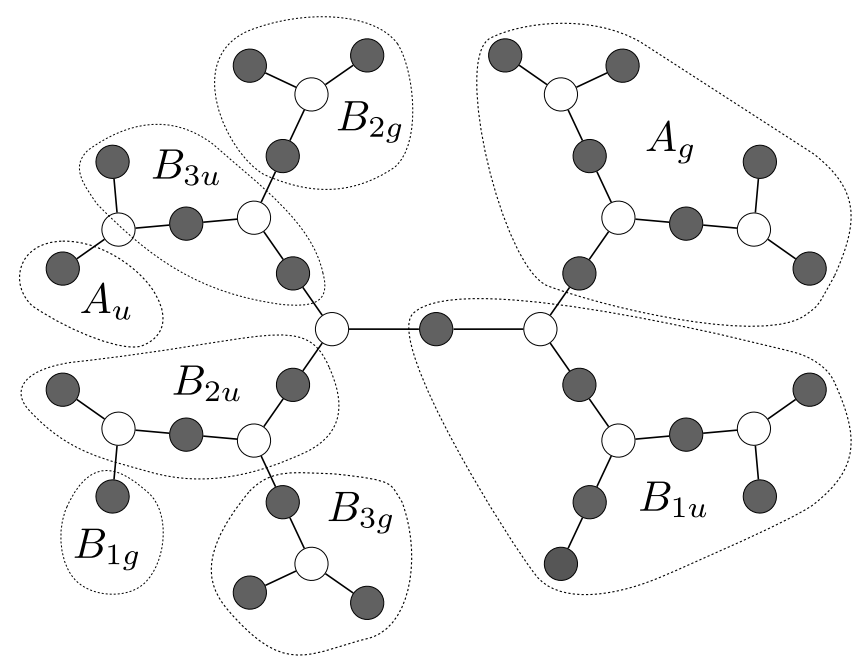

Figure S2: Tree-shaped network for $\mathrm{N}_{2}$ in the cc-pVDZ basis (14e, 28). The orbitals belonging to the same irreducible representation are grouped. $\mathrm{N}_{2}$ belongs to the $D_{2 h}$ point group. $A_{g}, A_{u}, B_{1 u}, B_{2 u}, B_{3 u}, B_{1 g}, B_{2 g}$ and $B_{3 g}$ are the Mulliken symbols of the irreducible representations of $D_{2 h}$. The orbitals closest to the Fermi level are put as close to the center as possible. Bonding and anti-bonding irreps are put close together.

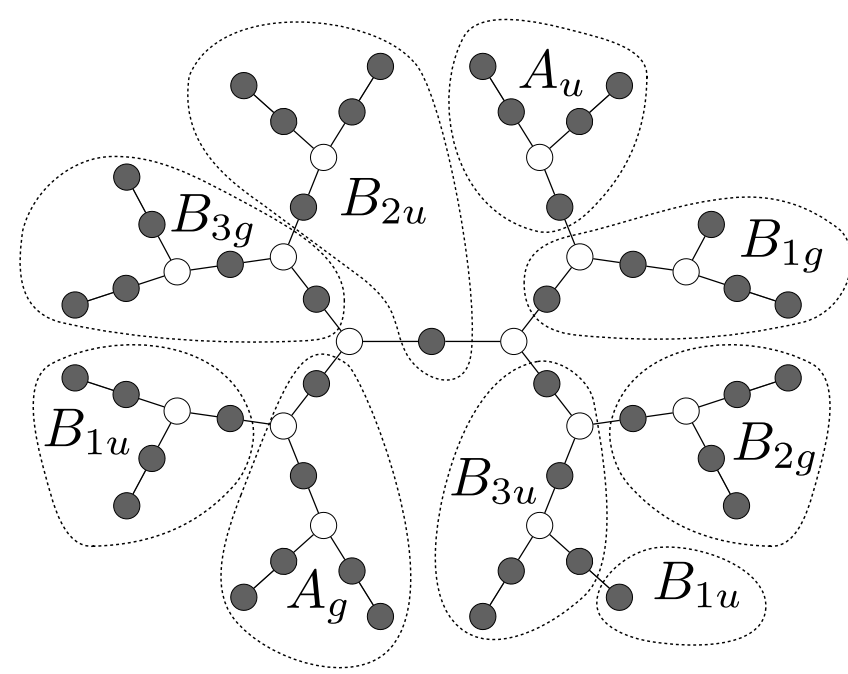

Figure S3: Tree-shaped network for $\left[\mathrm{Cu}_{2} \mathrm{O}_{2}\right]^{2+}$ in the $(26 \mathrm{e}, 44)$ active space for both isomers. The orbitals belonging to the same irreducible representation are grouped. $\left[\mathrm{Cu}_{2} \mathrm{O}_{2}\right]^{2+}$ belongs to the $D_{2 h}$ point group. $A_{g}, A_{u}, B_{1 u}, B_{2 u}, B_{3 u}, B_{1 g}, B_{2 g}$ and $B_{3 g}$ are the Mulliken symbols of the irreducible representations of $D_{2 h}$. The orbitals with the highest single-orbital entropy are put as close to the center as possible (entropies obtained from figs. 2 and 11 of ref. 33). Bonding and anti-bonding irreps are put close together. 